\title{
Measurement of the W Boson Mass at LEP2
}

\section{Jeremy Nowell}

Blackett Laboratory, Imperial College, London SW7 $2 B Z$

Jeremy. Noweilecern.ch *

ABSTRACT: The measurement of the mass of the $\mathrm{W}$ boson at the LEP2 collider is described, in particular the technique of direct reconstruction. Preliminary results are presented using data collected at centre of mass energies up to $202 \mathrm{GeV}$.

\section{Introduction}

\subsection{Motivation}

The mass of the $\mathrm{W}$ boson is a central component of the Standard Model of particle physics. It can be determined indirectly by a global fit to electroweak data using the following relationship:

$$
M_{\mathrm{W}}^{2}\left(1-\frac{M_{\mathrm{W}}^{2}}{M_{\mathrm{Z}}^{2}}\right)=\frac{\pi \alpha}{G_{F} \sqrt{2}} \cdot \frac{1}{1-\Delta r},
$$

where $\Delta r$ includes the effect of higher order corrections depending logarithmically on the mass of the Higgs boson and quadratically on the mass of the top quark. The current indirect measurement of the $\mathrm{W}$ mass has an uncertainty of $26 \mathrm{MeV} / c^{2}\left[\begin{array}{l}1 \\ 1\end{array}\right]$. A major goal of the LEP2 physics programme is to make a direct measurement of the $\mathrm{W}$ mass to a similar level of precision as the indirect measurement, thus providing a test of the Standard Model and allowing the Higgs mass to be constrained through the effect of the radiative corrections.

\subsection{W Pair Production at LEP2}

At LEP2 pairs of $\mathrm{W}$ bosons are produced by electron positron annihilation, proceeding at tree level through the CC03 diagrams, as shown in Figure $\bar{I}_{r}^{\prime}$ The first two of these diagrams are the annihilation diagrams, with either a $\mathrm{Z}^{0}$ boson or a photon produced in the $s$-channel, while the third diagram is the conversion diagram with the exchange of a neutrino in the $t$-channel. Each

\footnotetext{
${ }^{*}$ On behalf of the ALEPH collaboration.
}

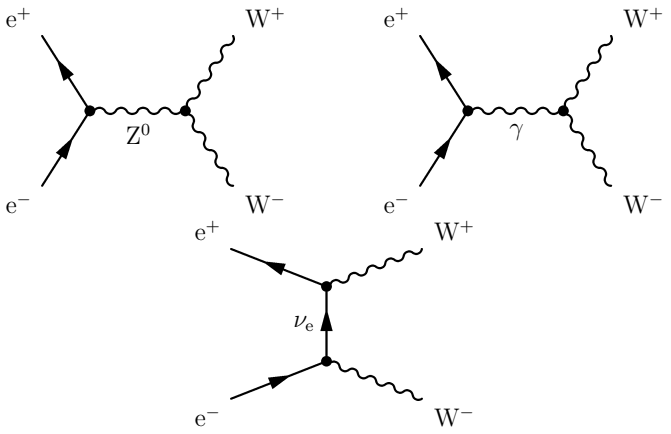

Figure 1: The CC03 diagrams of W pair production.

$\mathrm{W}$ then decays to either a quark and antiquark or lepton and associated neutrino. There are thus three distinct decay channels; fully-hadronic, semileptonic and fully-leptonic, with branching ratios as shown in Table 1 i.".

\begin{tabular}{|c|c|}
\hline $\begin{array}{c}\text { Decay } \\
\text { Channel }\end{array}$ & $\begin{array}{c}\text { Approximate } \\
\text { Branching Ratio }\end{array}$ \\
\hline $\mathrm{W}^{+} \mathrm{W}^{-} \rightarrow \mathrm{q} \overline{\mathrm{q}} \mathrm{q} \overline{\mathrm{q}}$ & $46 \%$ \\
\hline $\mathrm{W}^{+} \mathrm{W}^{-} \rightarrow \ell \nu \mathrm{q} \overline{\mathrm{q}}$ & $44 \%$ \\
\hline $\mathrm{W}^{+} \mathrm{W}^{-} \rightarrow \ell \nu \ell \nu$ & $10 \%$ \\
\hline
\end{tabular}

Table 1: The three $\mathrm{W}^{+} \mathrm{W}^{-}$decay channels.

\subsection{LEP2 Programme}

The LEP2 physics programme started in 1996 when the centre of mass energy of the collider was increased from the $\mathrm{Z}^{0}$ resonance past the threshold for $\mathrm{W}$ pair production, $161 \mathrm{GeV}$. Although only a small amount of data was collected at this luminosity, about $10 \mathrm{pb}^{-1}$ per experiment, a 
measurement of the $\mathrm{W}$ mass was made using the sensitivity of the WW cross section at this energy to the $\mathrm{W}$ mass [2]. Since this time the energy of the collider has been steadily increased to a value of $202 \mathrm{GeV}$ at the end of the 1999 data taking period. Each of the four LEP experiments, ALEPH, DELPHI, L3 and OPAL has collected approximately $450 \mathrm{pb}^{-1}$ of data above the WW threshold upto the end of 1999, corresponding to over $6000 \mathrm{WW}$ events per experiment.

\section{W Mass Measurement}

The four LEP collaborations have used, to one degree or another, differing techniques to measure the $\mathrm{W}$ mass at LEP2. In the short space available here only a brief overview can therefore be given. More details can be found in Refer-

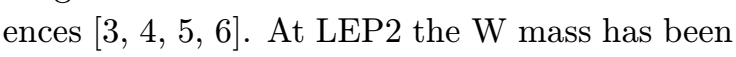
measured at all energies above the threshold using the technique of direct reconstruction. The general procedure is as follows:

- Select WW events.

- Reconstruct W's from decay products.

- Form invariant mass distribution.

- Extract W mass from this distribution.

Each of these steps will now be described in more detail.

\subsection{Event Selection}

The event selection depends on the particular decay channel being considered. The fully-leptonic channel is characterised by two highly energetic, acoplanar leptons. The presence of the two neutrinos means that fully-leptonic events have a large amount of missing energy and momentum. The main backgrounds to this channel are $Z^{0} Z^{0}$ production, $\mathrm{Z}^{0} \mathrm{e}^{+} \mathrm{e}^{-}$production and two photon events. Efficiencies achieved are typically between $40 \%$ and $60 \%$ with purities around $90 \%$.

Semi-leptonic events have two hadronic jets of particles and one isolated, energetic lepton together with missing energy and momentum due to the neutrino. The main backgrounds to semileptonic events are other four-fermion events such as $\mathrm{e}^{+} \mathrm{e}^{-} \rightarrow$ We $\nu$ and quark-antiquark pair production. Efficiencies are between $60 \%$ and $80 \%$ with purities between $85 \%$ and $95 \%$.

Fully-hadronic events have at least four jets with little missing energy and momentum. The main background to these events is the QCD background $\mathrm{e}^{+} \mathrm{e}^{-} \rightarrow \mathrm{q} \overline{\mathrm{q}}(\gamma)$. The complexity of these events and the difficulty in separating signal and background means that multivariate selection methods such as neural networks are widely used. Variables used in these algorithms are based on the event topology and the properties of the individual jets. Typical efficiencies are around $85 \%$ with purities around $90 \%$.

\subsection{Invariant Mass Reconstruction}

Once $\mathrm{W}$ pair candidate events have been selected the next step is to try and reconstruct the individual $\mathrm{W}$ bosons from their decay products and reconstruct their invariant mass.

The first step in this process is jet clustering. In semi-leptonic events the lepton is identified and the rest of the particles are forced into two jets, while in fully-hadronic events the particles are forced into four jets. Some collaborations also allow the possibility of five jets in fully-hadronic events since there is a reasonable chance of hard gluon radiation taking place.

After jet clustering a kinematic fit is applied to the event, significantly improving the invariant mass resolution. Since LEP is an electronpositron collider the centre of mass energy and momentum are known with a high degree of precision. The kinematic fit exploits this knowledge by varying the energy and momentum of the final state particles in order to satisfy the energy and momentum constraints:

$$
\sum_{i=1}^{N}\left(E_{i}, \mathbf{p}_{i}\right)=(\sqrt{s}, \mathbf{0}),
$$

where the sum is carried out over the final state particles, i.e. the lepton and jets, and $\sqrt{s}$ is the LEP centre of mass energy. The additional constraint of equal $\mathrm{W}$ boson masses may also be applied in the kinematic fit to improve further the invariant mass resolution.

The fully-hadronic channel has the additional problem of jet pairing. The four jets in the event 
may be paired to form the $\mathrm{W}$ bosons in three different ways, as shown in Table 2 . Several different methods have been used to select the optimum combination, including choosing the combination with the largest $\mathrm{CC} 03$ matrix element squared, or the combination with the minimum kinematic fit $\chi^{2}$ value. Alternatively all combinations may be used.

\begin{tabular}{|c|c|c|}
\hline Combination & $\mathrm{W}_{1}$ & $\mathrm{~W}_{2}$ \\
\hline 1 & $J_{1}+J_{2}$ & $J_{3}+J_{4}$ \\
\hline 2 & $J_{1}+J_{3}$ & $J_{2}+J_{4}$ \\
\hline 3 & $J_{1}+J_{4}$ & $J_{2}+J_{3}$ \\
\hline
\end{tabular}

Table 2: The different ways of pairing four jets.

After the kinematic fit and jet pairing the invariant mass may be formed:

$$
M_{\mathrm{W}}^{2}=\left(\sum_{i=1}^{n} E_{i}\right)^{2}-\left(\sum_{i=1}^{n} \mathbf{p}_{i}\right)^{2},
$$

where the sum is carried out over all particles assigned to the $\mathrm{W}$ being considered. It is from the distribution of the invariant mass, together with other mass related variables that the Wmass may be extracted.

\subsection{W Mass Extraction}

Several possibilities exist for the extraction of the $\mathrm{W}$ mass from the invariant mass distribution, however the most widely used at LEP2 and the one that will be described here is the technique of Monte Carlo reweighting. The other main method of mass extraction that is used is

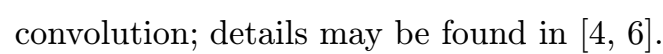

The principle behind Monte Carlo reweighting is to fit the Monte Carlo simulation to the data. The ideal scenario would be to generate fully simulated Monte Carlo events with many different input $\mathrm{W}$ masses, and perform the full reconstruction process on these events in the same way as for the data. The reconstructed mass distributions for the Monte Carlo could then be compared with that obtained from the data, with the measured $\mathrm{W}$ mass given by the value used as input to the Monte Carlo which best fits the data. The major difficulty with this procedure and the one that stops it being used is the computation time needed to generate the many millions

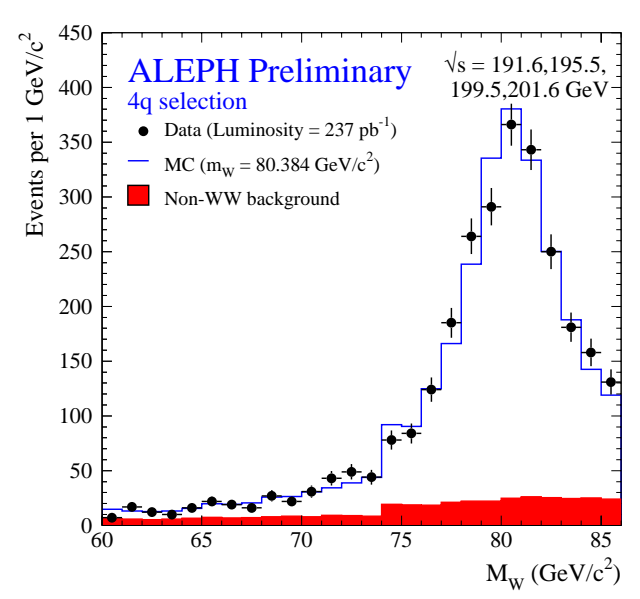

Figure 2: The invariant mass distribution for the fully hadronic decay channel from the ALEPH experiment.

of Monte Carlo events that are needed. The solution to the problem is to generate a large number of events at a given reference mass, $M_{\mathrm{W}}^{r e f}$, and then construct the invariant mass distribution. This distribution can be made to correspond to a different $\mathrm{W}$ mass, $M_{\mathrm{W}}^{0}$, by reweighting using the ratio of the $\mathrm{CCO} 3$ matrix elements squared:

$$
\begin{aligned}
& w_{i}\left(M_{\mathrm{W}}^{0}, \Gamma_{\mathrm{W}}^{r e f}\right)= \\
& \frac{\left|\mathcal{M}_{C C 03}\left(M_{\mathrm{W}}^{0}, \Gamma_{\mathrm{W}}^{r e f}, p_{i}^{1}, p_{i}^{2}, p_{i}^{3}, p_{i}^{4}\right)\right|^{2}}{\left|\mathcal{M}_{C C 03}\left(M_{\mathrm{W}}^{r e f}, \Gamma_{\mathrm{W}}^{r e f}, p_{i}^{1}, p_{i}^{2}, p_{i}^{3}, p_{i}^{4}\right)\right|^{2}}
\end{aligned}
$$

The series of distributions obtained in this way can be fitted to the data distribution to obtain the fitted $\mathrm{W}$ mass. The advantage of this technique is that the data and Monte Carlo are treated in exactly the same way so that there is no bias to correct for. Figure 2 in shows an example of the invariant mass distributions for the fully hadronic decay channel.

\section{Systematic Uncertainties}

The interesting stage has now been reached where the total systematic uncertainty on the measured $\mathrm{W}$ mass is of the same order as the statistical uncertainty for the combined LEP measurement. For this reason much work is ongoing to try and 
gain a better understanding of the major systematic uncertainties. The systematic uncertainties may be divided into two categories, those that are taken as correlated between experiments and those that are uncorrelated.

The uncertainties which are uncorrelated between experiments include background contamination in the data sample and effects related to the detectors such as uncertainties in the calorimeter energy scale. The uncertainty due to background contamination is typically small and that due to detector related effects is $\sim 10 \mathrm{MeV} / c^{2}$.

Correlated uncertainites include the uncertainties due to the modelling of the initial state radiation and fragmentation in the Monte Carlo. The latter is currently the largest systematic uncertainty on the LEP combined W mass measurement since it is taken as fully correlated between all decay channels and all experiments. It is determined by comparing the results obtained using different fragmentation models, such as those

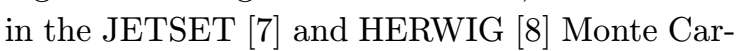
los, and is approximately $30 \mathrm{MeV} / c^{2}$.

Due to its use in the kinematic fit the uncertainty due to the measurement of the LEP beam energy, $E_{\text {beam }}$, translates directly into an uncertainty on the measured $\mathrm{W}$ mass:

$$
\Delta M_{\mathrm{W}}=M_{\mathrm{W}} \frac{\Delta E_{\text {beam }}}{E_{\text {beam }}} .
$$

In 1998 the uncertainty on the beam energy was $20 \mathrm{MeV}$, translating into an uncertainty on the $\mathrm{W}$ mass of $17 \mathrm{MeV} / c^{2}$.

There are two sources of possible systematic uncertainty which affect the fully-hadronic decay channel only. These are the final state interactions (FSI); colour reconnection and BoseEinstein effects. The decay of the two $\mathrm{W}$ bosons takes place over a much shorter space time distance, $\sim 0.1 \mathrm{fm}$, than the hadronisation scale, $\sim 1 \mathrm{fm}$. Therefore it can be expected that the hadronisation of each $\mathrm{W}$ is not independent, with possible colour strings being formed between the partons from different W's, leading to a possible shift in the reconstructed mass. It is predicted that Bose-Einstein effects will give an enhancement in the number of identical pions which are produced in the decay of the two $\mathrm{W}$ bosons, again leading to a possible shift in the reconstructed mass. The size of these possible uncertainties is estimated using phenomenological models which give a range of different values for the shift in the measured $\mathrm{W}$ mass. The largest shift is quoted as the uncertainty on the $\mathrm{W}$ mass, and this is $\sim 30 \mathrm{MeV} / c^{2}$ for each effect.

\section{Results}

The results presented here are preliminary, and are taken from the LEP Electroweak Working Group combination based on results presented at the winter 2000 conferences $[\overline{9} \bar{n}$. Figure $\overline{3}$, shows the combination of $\mathrm{W}$ mass measurements using direct reconstruction from the four LEP experiments which, when combined with the threshold measurement [2], gives a value of

$$
\begin{aligned}
M_{\mathrm{W}}= & 80.401 \pm 0.027(\text { stat }) \pm 0.032(\text { syst }) \\
& \pm 0.017(F S I) \pm 0.017(L E P) \mathrm{GeV} / c^{2},
\end{aligned}
$$

The combination of this result with measurements made at proton-antiproton colliders $\left[1 \overline{1} 0_{1}^{-}\right]$, gives a preliminary world average $\mathrm{W}$ mass of

$$
M_{\mathrm{W}}=80.419 \pm 0.038 \mathrm{GeV} / c^{2} .
$$

The comparison of the direct measurement and the indirect measurement is shown in Figure where the dependence on the Higgs and top quark masses is shown. This figure shows that the two measurements are in excellent agreement, and that a light value of the Higgs mass within the Standard Model is preferred.

\section{Summary}

An excellent performance of the LEP collider has allowed the four experiments to produce a preliminary measurement of the $\mathrm{W}$ boson mass of

$$
M_{\mathrm{W}}=80.401 \pm 0.048 \mathrm{GeV} / c^{2} .
$$

The statistical uncertainty on this measurement will be reduced further by the data collected during the year 2000, making further study of the systematic uncertainties very important. The direct and indirect measurements of the $\mathrm{W}$ boson mass are in excellent agreement, showing that the Standard Model is still strong, and a light Standard Model Higgs mass is indicated. 


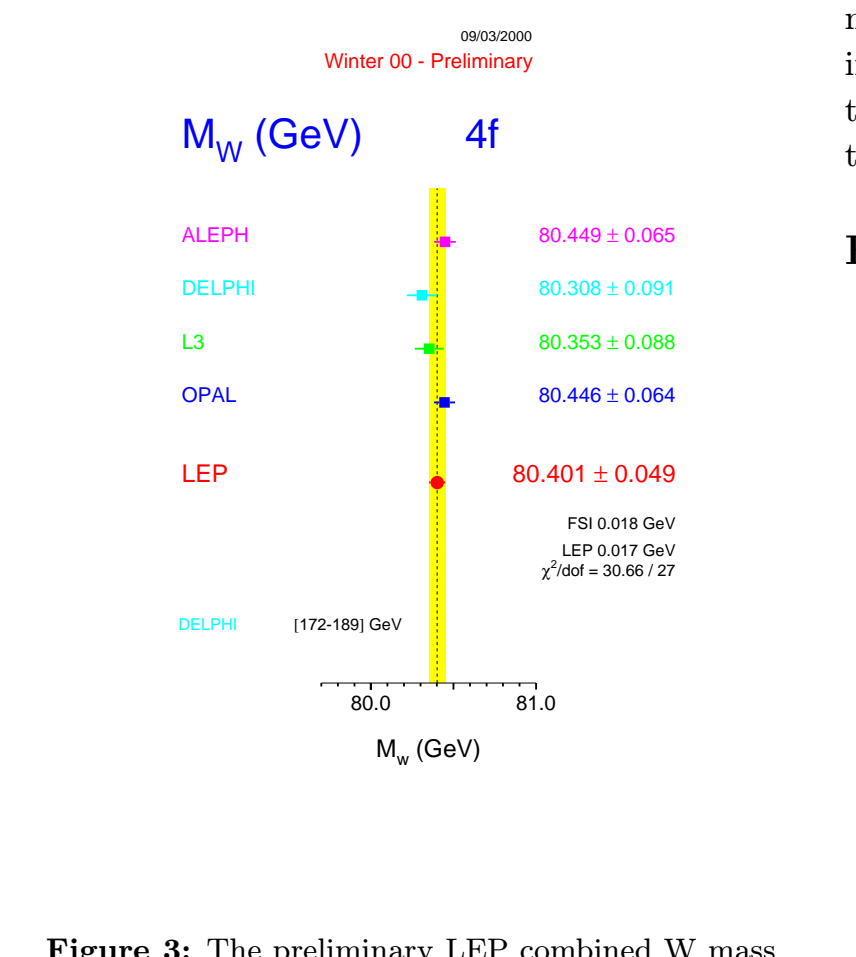

measurement using the method of direct reconstruction.

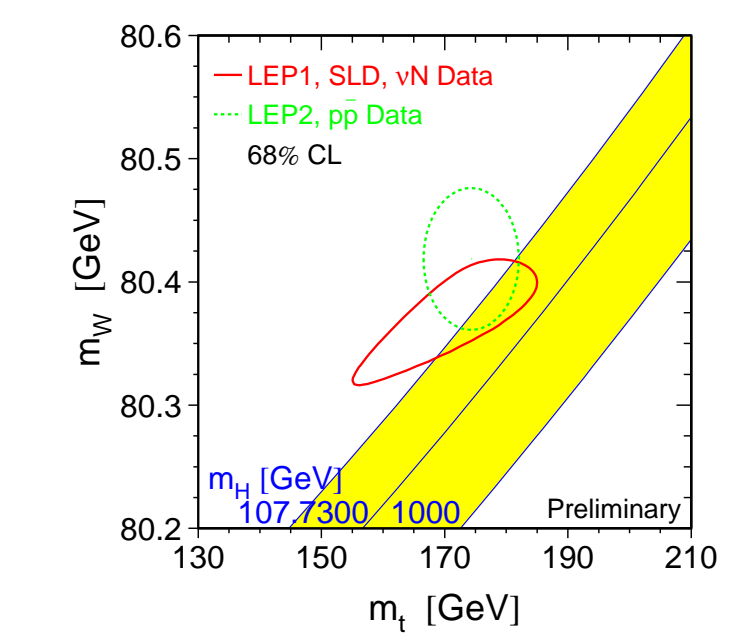

Figure 4: A comparison of the direct and indirect $\mathrm{W}$ mass measurements. The measurements are in excellent agreement and show that in the context of the Standard Model a light Higgs boson is preferred.

\section{Acknowledgments}

I would like to thank the organisers for inviting me to take part in an enjoyable conference in very interesting surroundings. I should especially like to thank Maria Victoria Arboleda for helping me to get home safely!

\section{References}

[1] The LEP Collaborations ALEPH, DELPHI, L3, OPAL, the LEP Electroweak Working Group and the SLD Heavy Flavour and Electroweak Groups, A Combination of Preliminary Electroweak Measurements and Constraints on the Standard Model, CERN-EP-2000-016.

[2] ALEPH Collaboration, 'Phys. Lett. B (1997) 347;

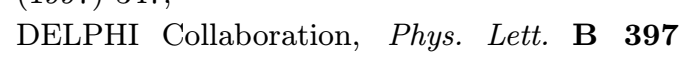
(1997) 158;

L3 Collaboration, iPhys. Lett. B 398 (1997) 223; OPAL Collaboration, IPhys. Lett. B $\mathbf{3 8 9}(1996)$ $4 \overline{1} \overline{6}_{\mathbf{r}}^{\prime \prime}$

[3] ALEPH Collaboration, 'Phys. Lett. B 422 (98)' 384! iPhys. Lett. B 453 (99) $121_{1}^{-}$Measurement of the $W$ Mass and Width in $\mathrm{e}^{+} \mathrm{e}^{-}$Collisions at 189 GeV CERN-EP-2000-045, submitted to Eur. Phys. J.C.

[4] DELPHI Collaboration, Eur. Phys. J. C2 (1998) 581; 'Phys. Lett. B 462 (1999) 410'

[5] L3 Collaboration, Phys. Lett. B 413 (1997) 176; 'Phys. Lett. B- 454 (1999) 386.

[6] OPAL Collaboration, Eur. Phys. J. C1 (1998) 395; 'Phys. Lett. B 453 (1999) 138, Measurement of the Mass and Width of the W Boson in $\mathrm{e}^{+} \mathrm{e}^{-}$Collisions at 189 GeV CERN-EP-2000099, hep-ex/0009018, submitted to Phys. Lett. B.

[7] Torbjorn Sjostrand, 'C'Comput. Phys. Commun.' $\mathbf{8 2}(1994)-74$.

[8] G. Marchesini et. al. iComput. Phys. Commun. $67(1992) 465:$ hep-ph/9607393.

[9] ALEPH Collaboration, ALEPH 2000-018 CONF 2000-015;

DELPHI Collaboration, DELPHI 2000-0147 CONF-446;

L3 Collaboration, L3 Note 2575;

OPAL Collaboration, OPAL PN422.

[10] CDF Collaboration, Measurement of the $W$ Boson Mass with the Collider Detector at Fermilab, hep-ex/0007044, submitted to Phys. Rev. D; D0 Collaboration, A New Measurement of the $W$ Boson Mass at D0, hep-ex/9907028.'. 